\title{
Anti-neoplastic effect of $\beta$-hydroxyisovalerylshikonin on a human choriocarcinoma cell line
}

\author{
NORIYUKI TAKAI, TAMI UEDA, MASAKAZU NISHIDA, KAEI NASU and HISASHI NARAHARA \\ Department of Obstetrics and Gynecology, Oita University Faculty of Medicine, Oita, Japan
}

Received January 15, 2010; Accepted March 15, 2010

DOI: $10.3892 / \mathrm{mmr} 00000290$

\begin{abstract}
HIVS), a compound isolated from the traditional asian medicinal herb Lithospermum radix, is an ATP non-competitive inhibitor of protein-tyrosine kinases such as v-Src and EGFR, and has been shown to induce apoptosis in several human tumor cell lines. We investigated the effect of $\beta$-HIVS in the choriocarcinoma cell line, BeWo. BeWo cells were treated with various concentrations of $\beta$-HIVS, and changes in cell growth, the cell cycle, apoptosis, and related parameters were examined. An MTT assay showed that BeWo cells were sensitive to the growth inhibitory effect of $\beta$-HIVS. Cell cycle analysis indicated that exposure to $\beta$-HIVS decreased the proportion of cells in the $S$ phase and increased the proportion in the $G_{0} / G_{1}$ phases of the cell cycle. Induction of apoptosis was confirmed by Annexin V staining of externalized phosphatidylserine and by the loss of mitochondrial transmembrane potential. This induction occurred in conjunction with the altered expression of genes related to cell growth, malignant phenotype, and apoptosis. These results suggest that $\beta$-HIVS may serve as a therapeutic agent for the treatment of choriocarcinoma.
\end{abstract}

\section{Introduction}

Gestational choriocarcinoma are a group of rare placenta disorders with varying potential for local or remote invasion in the form of metastases. Women with gestational choriocarcinoma who do not respond to well-established first-line chemotherapy have an extremely poor prognosis, even with the administration of multi-agent chemotherapy (1-3). Consequently, newer cytotoxic agents that may serve as definitive second- and third-line chemotherapy regimens are of interest.

The naphthoquinone pigment shikonin, the enantiomer of alkannin, has multiple pharmacological actions including

Correspondence to: Dr Noriyuki Takai, Department of Obstetrics and Gynecology, Oita University Faculty of Medicine, 1-1 Idaigaoka, Hasama-machi, Yufu-shi, Oita 879-5593, Japan

E-mail: takai@med.oita-u.ac.jp

Key words: EGFR, cell cycle, apoptosis, choriocarcinoma anti-bacterial, anti-fungal, anti-inflammatory, anti-thrombotic, anti-tumor, anti-gonadotropic and anti-human immunodeficiency virus activities (4). Extracts of the roots of Lithospermum radix, which contain shikonin and its various derivatives, were used in ancient Japan for the preparation of ointments for the treatment of cuts and burns, and were also ingested internally as an antidote to various poisons and as antipyretic and anti-inflammatory agents. $\beta$-hydroxyisovalerylshikonin ( $\beta$-HIVS) (Fig. 1), one of the derivatives of shikonin, is an ATP non-competitive inhibitor of the protein-tyrosine kinases (5), and has the strongest apoptosis-inducing activity of the shikonin derivatives (6). $\beta$-HIVS has been demonstrated to inhibit the proliferation and induce the apoptosis of tumor cells, including leukaemia (6,7), malignant melanoma (6), lung cancer $(5,7,8)$, endometrial and ovarian cancer (9) cells, at $\mathrm{IC}_{50}$ values ranging between $10^{-6}$ and $10^{-8} \mathrm{M}$. However, $\beta$-HIVS alone was shown to have no significant effect on the growth of human epidermoid carcinoma A431 cells at a concentration of $10^{-5} \mathrm{M}(10)$.

The effect of $\beta$-HIVS on choriocarcinoma cells has not previously been described. Therefore, this study was designed to determine the biological and therapeutic effects of $\beta$-HIVS in the BeWo choriocarcinoma cell line by examining whether the compound mediated the inhibition of cell growth, cell cycle arrest, apoptosis, and the expression of genes related to the malignant phenotype in these cells.

\section{Materials and methods}

Cell line. BeWo human choriocarcinoma cells were obtained from Riken Cell Bank (Ibaraki, Japan) and maintained as monolayers at $37^{\circ} \mathrm{C}$ in $5 \% \mathrm{CO}_{2}$ /air in HamF12 (Gibco, Rockville, MD, USA) containing $10 \%$ heat-inactivated fetal bovine serum (FBS) (Omega, Tarzana, CA, USA).

Chemicals. $\beta$-HIVS was obtained from Nagara Science (Gifu, Japan) and dissolved in ethanol at a concentration of $10^{-2} \mathrm{M}$ for use as a stock solution.

MTT assay. 3-(4,5-dimethylthiazol-2-yl)-2,5-diphenyltetrazolium bromide (MTT; Sigma) was dissolved in phosphate-buffered saline (PBS; $5 \mathrm{mg} / \mathrm{ml}$ ) and used to measure cellular proliferation. Cells $\left(1 \times 10^{3}\right)$ were incubated with $100 \mu$ of culture medium for $72 \mathrm{~h}$ in 96 -well plates, followed by the addition of $10 \mu \mathrm{l}$ MTT solution. After a further $4 \mathrm{~h}$ of incubation, solu- 


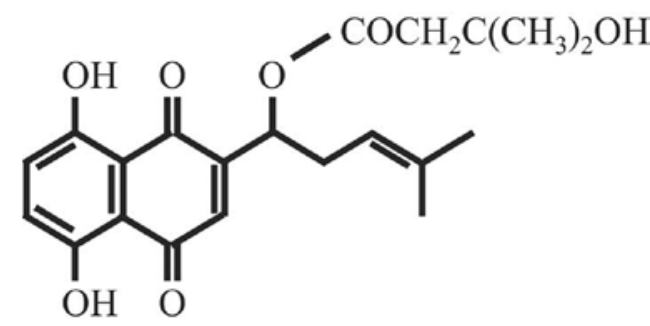

Figure 1. The chemical structure of $\beta$-HIVS.

bilization solution ( $50 \mu \mathrm{l}$ of $20 \%$ SDS) was added, then cells were incubated at $37^{\circ} \mathrm{C}$ for $16 \mathrm{~h}$. In this assay, MTT is cleaved to an orange formazan dye by metabolically active cells. The dye was directly quantified using an enzyme-linked immunosorbent assay reader at $540 \mathrm{~nm}$.

Cell cycle analysis by flow cytometry. The cell cycle was analyzed by flow cytometry after 3 days of culturing as previously described (11). Cells $\left(5 \times 10^{4}\right)$ were exposed to $\beta$-HIVS in 6 -well flat-bottomed plates for $72 \mathrm{~h}$. Analysis was performed immediately after staining using the CELLFit program (Becton Dickinson, San Jose, CA, USA), whereby the S phase was calculated using an RFit model.

Measurement of apoptosis by flow cytometry with Annexin V/ propidium iodide. Cells were plated and grown overnight until they reached $80 \%$ confluence, and then treated with $\beta$-HIVS. After 72 h, detached cells in the medium were collected, and the remaining adherent cells were harvested by trypsinization. The cells $\left(1 \times 10^{5}\right)$ were washed with PBS and resuspended in $250 \mu \mathrm{l}$ binding buffer (Annexin V-FITC kit; Becton Dickinson) containing $10 \mu \mathrm{l}$ of $20 \mu \mathrm{g} / \mathrm{ml}$ propidium iodide (PI) and $5 \mu \mathrm{l}$ of Annexin V-FITC, which binds to phosphatidylserine translocated to the exterior of the cell membrane early in the apoptosis pathway as well as during necrosis. After incubation for $10 \mathrm{~min}$ at room temperature in a light-protected area, the samples were analyzed on a FACSCalibur flow cytometer (Becton Dickinson). FITC and PI emissions were detected in the FL-1 and FL-2 channels, respectively. For each sample, data from 30,000 cells were recorded in list mode on logarithmic scales. Subsequent analysis was performed with CellQuest software (Becton Dickinson).

Mitochondrial transmembrane potential. Cells were prepared for FACS as described above and stained as previously described (12) using the Mitocapture Apoptosis Detection kit (Biovision, Palo Alto, CA, USA) according to the manufacturer's instructions with a fluorescent lipophilic cationic reagent that assesses mitochondrial membrane permeability.

Western blot analysis. Cells were washed twice in PBS, suspended in lysis buffer [50 mM Tris (pH 8.0), $150 \mathrm{mM}$ $\mathrm{NaCl}, 0.1 \%$ SDS, $0.5 \%$ sodium deoxycholate, $1 \% \mathrm{NP} 40$, phenylmethylsulfonyl fluoride at $100 \mu \mathrm{g} / \mathrm{ml}$, aprotinin at $2 \mu \mathrm{g} / \mathrm{ml}$, pepstatin at $1 \mu \mathrm{g} / \mathrm{ml}$ and leupeptin at $10 \mu \mathrm{g} / \mathrm{ml}]$, and placed on ice for $30 \mathrm{~min}$. After centrifugation at $15,000 \mathrm{x} \mathrm{g}$ for 15 min at $4^{\circ} \mathrm{C}$, the suspension was collected. Protein concentrations were quantified using the Bio-Rad protein Assay Dye

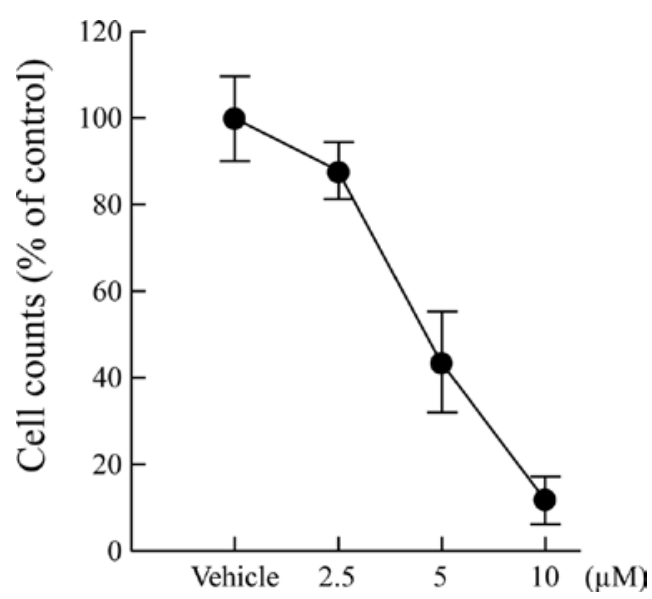

Figure 2. Effect of $\beta$-HIVS on the growth of human choriocarcinoma cells in vitro. BeWo choriocarcinoma cells were treated with either $\beta$-HIVS at various concentrations $\left(2.5 \times 10^{-6}-1 \times 10^{-5} \mathrm{M}\right)$ or the dilutant (control) for $48 \mathrm{~h}$, and growth (\% of control) was measured using an MTT assay. Results represent the means \pm SD of three independent experiments with dishes in triplicate.

Reagent Concentrate (Bio-Rad Laboratories, Hercules, CA, USA) according to the manufacturer's instructions. Whole cell lysates $(40 \mu \mathrm{g})$ were resolved by SDS-PAGE on a $4-15 \%$ gel, transferred to a polyvinylidene difluoride membrane (Immobilon; Amersham, Arlington Heights, IL, USA) and sequentially probed with antibodies against p21 ${ }^{\mathrm{WAF} 1}(1: 1,000$, Ab-1; Oncogene, San Diego, CA, USA), cyclin A (1:1,000; BD Pharmingen), cyclin D1 (1:1,000; BD Pharmingen), bcl-2 (1:1,000; BD Pharmingen), cleaved caspase-9 (1:1,000; BD Pharmingen) and GAPDH mAb (1:10,000; Research Diagnostics, Flanders, NJ, USA). The blots were developed using the enhanced chemiluminescent (ECL) kit (Amersham).

Statistical analysis. Numerical data were expressed as the means \pm SD. Significance was determined by conducting a paired Student's t-test.

\section{Results}

Effect of $\beta$-HIVS on the proliferation and viability of choriocarcinoma cells. The antitumor effects of $\beta$-HIVS on the choriocarcinoma cells were examined in vitro using an MTT assay with a 2-day exposure to $\beta$-HIVS (Fig. 2). At $4.5 \times 10^{-6} \mathrm{M}$ $\beta$-HIVS, BeWo choriocarcinoma cells showed significant sensitivity to the compound, with a $50 \%$ inhibition $\left(\mathrm{ED}_{50}\right)$ of growth.

Effect of $\beta$-HIVS on the cell cycle in choriocarcinoma cells. We next investigated whether $\beta$-HIVS led to the induction of apoptosis and/or cell cycle arrest in the choriocarcinoma cells. $\beta$-HIVS led to a dramatic increase in the sub $G_{0} / G_{1}$ apoptotic cell population compared to treatment with vehicle alone, with a concomitant decrease in the proportion of cells in the $\mathrm{S}$ phase (Table I).

EFfect of $\beta$-HIVS on the apoptosis of choriocarcinoma cells. To assess the ability of BeWo choriocarcinoma cells to undergo apoptosis in response to $\beta$-HIVS exposure, and to help distinguish between the different types of cell death, 
Table I. Results of cell cycle analysis in the BeWo choriocarcinoma cell line.

\begin{tabular}{lcc}
\hline & Vehicle & $\beta$-HIVS $\left(5 \times 10^{-6} \mathrm{M}\right)$ \\
\hline Sub G $_{0} / \mathrm{G}_{1}(\%)$ & $1 \pm 0$ & $22 \pm 7$ \\
$\mathrm{G}_{0} / \mathrm{G}_{1}(\%)$ & $40 \pm 9$ & $49 \pm 11$ \\
$\mathrm{~S}(\%)$ & $37 \pm 8$ & $10 \pm 2$ \\
$\mathrm{G}_{2} / \mathrm{M}(\%)$ & $21 \pm 5$ & $19 \pm 9$ \\
\hline
\end{tabular}

Table II. Cell death measured by Annexin V and MTP and detected by flow cytometry in the BeWo choriocarcinoma cell line.

Vehicle $\quad \beta$-HIVS $\left(5 \times 10^{-6} \mathrm{M}\right)$

\begin{tabular}{lcl}
\hline Annexin V assay & & \\
Viable (LL) (\%) & $95 \pm 1$ & $70 \pm 15$ \\
Apoptosis (LR) (\%) & $2 \pm 0$ & $16 \pm 7$ \\
Necrosis (UR) (\%) & $3 \pm 1$ & $12 \pm 5$ \\
MTP assay & & \\
Viable (\%) & $95 \pm 2$ & $71 \pm 9$ \\
Apoptosis (\%) & $5 \pm 1$ & $29 \pm 3$ \\
\hline
\end{tabular}

we double-stained $\beta$-HIVS-treated cells with Annexin V and PI and analyzed the results using flow cytometry. Annexin V binding combined with PI labeling was performed for the distinction of early apoptotic (Annexin $\mathrm{V}^{+} / \mathrm{PI}$ ) and necrotic (Annexin $\mathrm{V}^{+} / \mathrm{PI}^{+}$) cells. At increasing doses of $\beta$-HIVS, we detected a simultaneous increase in both the Annexin $\mathrm{V}^{+}$ PI- fraction (early apoptotic) and Annexin $\mathrm{V}^{+} / \mathrm{PI}^{+}$(regarded as necrotic) subpopulations (Table II).

Effect of $\beta$-HIVS on mitochondrial transmembrane potential in choriocarcinoma cells. Loss of MTP has been shown to occur prior to nuclear condensation and caspase activation, and is linked to cytochrome $c$ release in many, but not all, apoptotic cells $(13,14)$. Using MitoCapture staining and flow cytometry, MMP was analyzed in BeWo choriocarcinoma cells treated with $\beta$-HIVS. Intracellular fluorescence was assayed by FACS staining after loading with an intramitochondrial dye. High fluorescence at $575 \mathrm{~nm}$ (FL2) corresponds to the aggregated form of the dye and is proportional to an intact MTP, whereas loss of MTP leads to a loss of 575 fluorescence and an increase in fluorescence at $525 \mathrm{~nm}$ (FL1; monomeric form of the dye). Untreated cells exhibit high 575 fluorescence, indicating normal MTP. Treatment of cells with $\beta$-HIVS results in the loss of 575 fluorescence and an increase in fluorescence at $525 \mathrm{~nm}$, indicating the loss of MTP (Table II).

Effect of $\beta$-HIVS on the expression of cell cycle- and apoptosis-related proteins. $\mathrm{p} 21^{\mathrm{WAF} 1}$ are cyclin-dependent kinase inhibitors (CDKI) that bind to cyclin-dependent kinase complexes and decrease kinase activity, and may thus act as key regulators of $\mathrm{G}_{0} / \mathrm{G}_{1}$ accumulation (reviewed in ref. 11). We

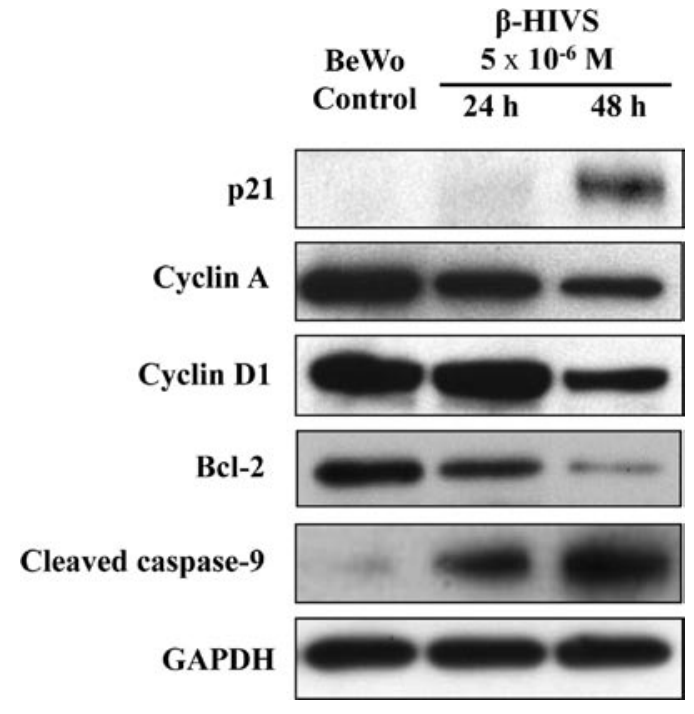

Figure 3. Cell cycle- and apoptosis-related protein expression in BeWo cells measured by Western blot analysis. BeWo cells were treated with $5 \times 10^{-6} \mathrm{M}$ $\beta$-HIVS, and cell lysates were harvested after 24 and $48 \mathrm{~h}$. Western blot analysis was performed with a series of antibodies. Control cells were treated with vehicle alone. The amount of protein was normalized by comparison to GAPDH levels.

examined the effect of $\beta$-HIVS on the expression of $\mathrm{p} 21^{\mathrm{WAF}}$ in the BeWo cell line using Western blot analysis (Fig. 3). $\beta$-HIVS markedly up-regulated the level of $\mathrm{p} 21^{\mathrm{WAF} 1}$ proteins, which were expressed at negligible levels in the untreated choriocarcinoma cells. Furthermore, $\beta$-HIVS decreased cyclin A and D1 levels in the BeWo cells (Fig. 3). The expression of bcl-2 was down-regulated following exposure to $\beta$-HIVS in the BeWo cells (Fig. 3). After treatment with $\beta$-HIVS, cleaved fragments of procaspase-9 were detected (Fig. 3), suggesting that the apoptosome pathway was activated by $\beta$-HIVS.

\section{Discussion}

Most of the PTK inhibitors studied to date, including STI571 (Gleevec), ZD1839 (Iressa), SU5416 and PD173074, have chemical structures that resemble the structure of ATP and thus compete with ATP for binding to the ATP binding site in the catalytic domain of the PTKs, with a resulting inhibition of enzymatic activity. In contrast to these ATP-competitive inhibitors of PTK, the shikonin derivative $\beta$-HIVS, isolated from the plant Lithospermum radix, inhibits the activity of $\mathrm{v}-\mathrm{Src}$ in an ATP-non-competitive manner (5). Since $\beta$-HIVS does not need to compete with ATP in the intracellular environment, it is very useful for the inhibition of PTK activity in vivo. $\beta$-HIVS binds to the promoter region of PLK1 and other $\beta$-dependent PTKs, inhibiting cyclin expression and promoting the function of cyclin-dependent kinase inhibitors (15).

The results of the present study indicate that $\beta$-HIVS is highly effective in suppressing the growth of $\beta$ choriocarcinoma cells at a low concentration $\left(10^{-6}-10^{-5} \mathrm{M}\right)$, in agreement with the findings of previous reports (5-8). The marked arrest of cancer cells in the $\mathrm{G}_{0} / \mathrm{G}_{1}$ phase of the cell cycle is likely to account for this effect. $\mathrm{p} 21^{\mathrm{WAF} 1}$ are cyclin-dependent kinase inhibitors that play an important role in the cell cycle by causing blockade at the $\mathrm{G}_{1}$ phase (16). The protein level of $\mathrm{p} 21^{\mathrm{WAF} 1}$ was increased 
in the BeWo cell line following treatment with $\beta$-HIVS, suggesting that the up-regulation of $\mathrm{p} 21^{\mathrm{WAF} 1}$ is a mechanism by which this agent inhibits choriocarcinoma cell growth.

The cyclins are known to be key proteins in the control of cell proliferation. Cyclin A acts from the late $\mathrm{G}_{1}$ phase through to the $\mathrm{M}$ phase of the cell cycle, forming a complex with cdk2 in the late $\mathrm{G}_{1}-\mathrm{S}$ phase and with cdc2 in the $\mathrm{G}_{2} / \mathrm{M}$ phase (17). $\beta$-HIVS decreased the expression of cyclin $A$, an effect that modulated the activity of the downstream $\mathrm{pRb} / \mathrm{E} 2 \mathrm{~F}$ axis, thereby triggering cell cycle arrest, especially in the $\mathrm{G}_{1}$ phase at higher concentrations. We showed that treatment with $\beta$-HIVS dramatically and significantly increased the number of apoptotic cells in the BeWo cell line. This effect was associated with a decrease in the levels of the anti-apoptotic protein bcl-2 and with an increase in the levels of cleaved caspase-9. It is possible that apoptotic signaling was induced by this down-regulation of bcl-2 expression and activation of caspase-9, thus the bcl-2-mediated cascades are potentially involved in $\beta$-HIVS-induced apoptosis.

In conclusion, this is the first report to demonstrate that $\beta$-HIVS exhibits antiproliferative activity, potently induces cell cycle arrest, and stimulates apoptosis in human choriocarcinoma cells. These events were accompanied by the induction of $\mathrm{p} 21^{\mathrm{WAFl}}$ and the down-regulation of several anti-apoptosis and cell cycle-related proteins, including bcl-2, cyclin A and cyclin D1. Although the safety of this agent in clinical practice has not been established, these findings suggest that $\beta$-HIVS may serve as a therapeutic agent in the treatment of choriocarcinoma. However, the present study involves preliminary in vitro experiments only, and in only one cell line; its findings must be tested in other cell lines and in animal models to confirm the benefits of $\beta$-HIVS for the treatment of choriocarcinoma.

\section{Acknowledgments}

This study was supported by a grant (project code FK344 to N.T.) from the Japan Society of Gynecologic Oncology, a Grant-in-Aid (No. 21592139 to N.T.) for Scientific Research from the Ministry of Education, Culture, Sports, Science and Technology of Japan, and a Research Fund at the Discretion of the President, Oita University.

\section{References}

1. Jones WB, Cardinale C and Lewis JL Jr: Management of the high-risk gestational trophoblastic disease: the Memorial Hospital experience. Int J Gynecol Cancer 7: 27-33, 1997.

2. Berkowitz RS, Goldstein DP and Bernstein MR: Modified triple chemotherapy in the management of high-risk gestational trophoblastic tumors. Gynecol Oncol 19: 173-181, 1984.

3. Surwit EA and Hammond CB: Treatment of metastatic trophoblastic disease with poor prognosis. Obstet Gynecol 55: 565-570, 1980.

4. Chen X, Yang L, Oppenheim JJ and Howard OMZ: Cellular pharmacology studies of shikonin derivatives. Phytother Res 16: 199-209, 2002.

5. Hashimoto S, Xu M, Masuda Y, et al: $\beta$-Hydroxyisovalerylshikonin is a novel and potent inhibitor of protein tyrosine kinases. Jpn J Cancer Res 93: 944-951, 2002.

6. Hashimoto S, Xu M, Masuda Y, et al: $\beta$-Hydroxyisovalerylshikonin inhibits the cell growth of various cancer cell lines and induced apoptosis in leukemia HL60 cells through a mechanism different from those of Fas and etoposide. J Biochem 125: 17-23, 1999.

7. Masuda Y, Shima G, Aiuchi T, et al: Involvement of tumor necrosis factor receptor-associated protein 1 (TRAP1) in apoptosis induced by $\beta$-hydroxyisovalerylshikonin. J Biol Chem 279: 42503-42515, 2004.

8. Xu Y, Kajimoto S, Nakajo S and Nakaya K: $\beta$-hydroxyisovalerylshikonin and cisplatin act synergistically to inhibit growth and to induce apoptosis of human lung cancer DMS114 cells via a tyrosine kinase-dependent pathway. Oncology 66: 67-75, 2004.

9. Takai N, Ueda T, Nishida M, Nasu K and Narahara H: Betahydroxyisovalerylshikonin has a profound anti-growth activity in human endometrial and ovarian cancer cells. Gynecol Oncol. 109: 107-114, 2008.

10. Xu Y, Nakajo S and Nakaya K: Synergistic inhibitory effects of transplatin and beta-hydroxyisovalerylshikon on carcinoma A431 cells involve epidermal growth factor receptor. Cancer Lett 188: 67-72, 2002.

11. Takai N, Desmond JC, Kumagai T, et al: Histone deacetylase inhibitors have a profound anti-growth activity in endometrial cancer cells. Clin Cancer Res 10: 1141-1149, 2004.

12. Takai N, Ueda T, Nishida M, Nasu K and Narahara H: Bufalin induces growth inhibition, cell cycle arrest and apoptosis in human endometrial and ovarian cancer cells. Int J Mol Med 21: 637-643, 2008.

13. Rimon G, Bazenet CE, Philpott KL and Rubin LL: Increased surface phosphatidylserine is an early marker of neuronal apoptosis. J Neurosci Res 48: 563-570, 1997.

14. Chen Y, Kramer DL, Diegelman P, Vujcic S and Porter CW: Apoptotic signaling in polyamine analogue-treated SK-MEL-28 human melanoma cells. Cancer Res 61: 6437-6444, 2001.

15. Masuda Y, Nishida A, Hori K, et al: $\beta$-Hydroxyisovalerylshikonin induces apoptosis in human leukemia cells by inhibiting the activity of a polo-like kinase 1 (PLK1). Oncogene 22: 1012-1023, 2003.

16. Johnson DG and Walker CL: Cyclins and cell cycle checkpoints. Annu Rev Pharmacol Toxicol 39: 295-312, 1999.

17. Sherr CJ: G1 phase progression: cyclin on cue. Cell 79: 551-555, 1994. 